\title{
Error estimation of a homogenized streamwise periodic boundary layer
}

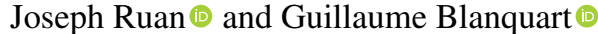 \\ California Institute of Technology, 1200 E California Blvd, Pasadena, California 91125, USA
}

(Received 5 March 2021; accepted 5 October 2021; published 29 November 2021)

\begin{abstract}
An analysis was conducted of the transpiration velocity of the streamwise periodic simulation of the turbulent flat plate boundary layer. As an often imposed quantity in numerical simulation, the transpiration velocity plays an important role in the shape of the wall-normal profile near the outer layer. Unlike other simulation frameworks which impose the transpiration velocity, the recently proposed framework [Ruan and Blanquart, Phys. Rev. Fluids 6, 024602 (2021)] relies on a single-scale rescaling of the wallnormal coordinate to perform streamwise periodic boundary layer simulations. The current manuscript highlights that any error in the transpiration velocity from these simulations is due to a difference in inner and outer layer growth rates. A new multiscale framework to compensate for these differing growth rates is proposed and verified but ultimately has negligible impact on the mean profiles and turbulent intensities. These remain in excellent agreement with previously published values. It is shown that any error in the mean continuity equation expresses itself primarily as an error in transpiration velocity, which decreases with Reynolds number. Overall, the error in the transpiration velocity can be used to quantify the error in single-scale, streamwise periodic simulations.
\end{abstract}

DOI: 10.1103/PhysRevFluids.6.114604

\section{INTRODUCTION}

Turbulent boundary layers have persistently maintained the interest of the fluids community at large due to the abundance of turbulent phenomena within the flow. From the uniform momentum zones [1], to the large-scale motions [2], to asymptotic Reynolds number behavior [3], the flat plate turbulent boundary layer has several unique features that unfortunately only fully emerge at high Reynolds number. To thoroughly investigate them from a computational perspective requires simulations at Reynolds numbers at least an order of magnitude greater than the current state-ofthe-art [4].

Recently, Ruan and Blanquart [5] provided a method for streamwise periodic direct numerical simulation (DNS) of boundary layers that reduces the required domain size by an order of magnitude. To do so, a single-scale wall-normal rescaling was applied to the boundary layer. The resulting simulations produced accurate global quantities and mean profiles. Alongside the domain reduction, the simulation framework also avoided imposing the transpiration velocity, which most growing boundary layer simulations require [6-8].

The transpiration velocity $\left(V_{\infty}\right)$ is an often overlooked boundary layer quantity. Because it is small compared to the free-stream velocity $\left(V_{\infty} \sim 0.005 U_{\infty}\right)$, it is difficult to resolve in experimental data and overall difficult to validate numerically. Nevertheless, it is directly related to the growth of the boundary layer and even the inner layer dynamics [9]. Specifically, integration of continuity immediately gives that $V_{\infty}=U_{\infty} d \delta^{*} / d x$, where $\delta^{*}$ is the displacement thickness. It has also been shown that near the free stream $\left(y \approx \delta_{99}\right)$, the mean wall-normal profile $V / V_{\infty}$ is independent of Reynolds number [9]. This fact was crucial in modeling the mean stress profiles in the outer layer [10]. Consequently, the transpiration velocity is crucial to the stream-wise development of skin 
friction coefficient and shape factor. In all, despite being relatively absent in many experimental databases, its presence is felt throughout key turbulent quantities and profiles.

The mean fields of turbulent boundary layers are two-dimensional and in order to conduct simulations, one needs to specify the stream-wise inlet, stream-wise outlet, and wall-normal outlet for numerical well-posedness. Although much of the current literature on boundary layer simulation has been focused on the inflow conditions [6-8,11-13], the majority of the stream-wise growing boundary layer simulations [6,7,11-13] impose a $V_{\infty}(x)$ profile at the top of the domain. As mentioned earlier, doing so is equivalent to imposing $d \delta^{*} / d x$. Consequently, the agreement of their other global quantities with empirical fits is only expected. However, the stream-wise periodic framework [5] imposed no such transpiration velocity, and yet the resultant global quantities of interest were as good as if not better than those of stream-wise growing simulations. These surprisingly good results form the basis for the present study. Since no transpiration velocity was imposed, the resultant transpiration velocity within the periodic simulation can be used as a quantification of numerical error. Given how deeply tied the transpiration velocity is to boundary layer dynamics, it is necessary to (1) investigate and quantify the error in transpiration velocity and (2) look at the impact of multi scale vs single-scale simulations for a range of Reynolds numbers numerically and analytically.

First, Sec. II gives a quick overview of Ref. [5] and theorizes how errors in the transpiration velocity might develop. Then we investigate the multiscale nature of the boundary layer, a priori in Sec. III and a posteriori in Sec. IV. Finally, Sec. V provides an interrogation of the continuity equation via an error budget.

\section{ANALYSIS OF STATIONARY BOUNDARY LAYER}

\section{A. A review of streamwise periodic boundary layer simulations}

We only provide a brief overview of the stream-wise periodic boundary layer framework. The reader is referred to Ruan and Blanquart [5] for a complete derivation of Eqs. (2)-(4) (below). A flat plate boundary layer under Cartesian coordinates $\left(x_{1}, x_{2}, x_{3}\right)$, has velocity components in the stream-wise $\left(x_{1}\right)$, wall-normal $\left(x_{2}\right)$, and span-wise $\left(x_{3}\right)$ directions, respectively. We define the following new coordinates through a wall-normal rescaling:

$$
\xi_{1}=x_{1}, \quad \xi_{2}=x_{2} \frac{q_{0}}{q\left(x_{1}\right)}, \quad \xi_{3}=x_{3},
$$

where $q=q(x)$ is a single length scale meant to parallelize the boundary layer locally. This coordinate change is applied to the Navier-Stokes equations. Two critical assumptions are further applied:

(1) There exists a function $q\left(x_{1}\right)$ such that ensemble-averaged quantities are both statistically stationary and statistically homogeneous in the $\xi_{1}, \xi_{3}$ directions.

(2) The rescaled governing equations are evaluated at the stream-wise location where $x_{1}=x_{0}$, $q\left(x_{1}\right)=q_{0}$ and are assumed to be valid for a narrow stream-wise domain centered at $x_{1}=x_{0}$.

After an a priori and a posteriori analyses, all terms with the second streamwise derivative $\left(q^{\prime \prime}\right)$ and the square of the first streamwise derivative $\left(q^{\prime 2}\right)$ are neglected, and the following equations are obtained:

$$
\begin{gathered}
\frac{\partial u_{j}}{\partial \xi_{j}}=\underbrace{\xi_{2} \frac{q^{\prime}}{q} \frac{\partial u_{1}}{\partial \xi_{2}}}_{\text {source term }}, \\
\frac{\partial u_{i}}{\partial t}=-u_{j} \frac{\partial u_{i}}{\partial \xi_{j}}-\frac{1}{\rho} \frac{\partial P}{\partial \xi_{i}}+v \frac{\partial^{2} u_{i}}{\partial \xi_{j}^{2}}+\underbrace{\xi_{2} \frac{q^{\prime}}{q} u_{1} \frac{\partial u_{i}}{\partial \xi_{2}}}_{\text {source term }} .
\end{gathered}
$$

The additional source terms within Eqs. (2) and (3) continuously rescale the boundary layer to account for its overall growth. In the regular/conventional spatially growing boundary layer, the 
Reynolds shear stress in the stream-wise momentum equation is primarily balanced by the normal stress term in the outer layer. Similarly, in Eq. (3), the Reynolds shear stress is likewise balanced by the source term in the outer layer. However, this source term is homogeneous in the stream-wise direction and thereby removes the stream-wise growth of the boundary layer.

Each of the source terms contain a ratio $q^{\prime} / q$ owing to the coordinate change, and the term requires closure. Assumption 2 implies that the closure equation for $q^{\prime} / q$ must simultaneously balance the time, span-wise, and stream-wise averaged continuity and momentum equations throughout the boundary layer. To satisfy this constraint, the following closure was directly derived from the wall-normal integrated mean continuity and stream-wise momentum equations:

$$
\frac{q^{\prime}}{q}=\frac{\tau_{w} / \rho}{\int_{0}^{\infty}\left(u_{1, \infty}\left\langle u_{1}\right\rangle_{\xi_{1}, \xi_{3}}-\left\langle u_{1} u_{1}\right\rangle_{\xi_{1}, \xi_{3}}\right) d \xi_{2}},
$$

where $\tau_{w}=\left.\rho v\left\langle\partial u_{1} / \partial \xi_{2}\right\rangle_{\xi_{1}, \xi_{3}}\right|_{\xi_{2}=0}$ is the wall shear stress, $\rho$ is the density, $v$ is the kinematic viscosity, $u_{1, \infty}$ is the free-stream velocity, and $\langle\cdot\rangle_{\xi_{1}, \xi_{3}}$ denotes averaging in the homogeneous stream-wise $\left(\xi_{1}\right)$ and span-wise $\left(\xi_{3}\right)$ directions. In practice, the simulation solves the governing equations with periodic stream-wise and span-wise directions, and the closure equation is calculated instant- aneously.

The solutions resulting from these simulations were found to produce global quantities in good agreement with established empirical and prior DNS values. The shape factor $\left(H_{12}=\delta^{*} / \theta\right)$ and skin-friction coefficient $\left(C_{f}=2 \tau_{w} / \rho u_{1, \infty}^{2}\right)$ were within $0.4 \%$ and $1.6 \%$ of empirical fits [3,14], respectively, where $\delta^{*}$ is the displacement thickness, and $\theta$ is the momentum thickness. These global quantities were comparable to those of stream-wise growing boundary layer simulations. It should be noted that these simulations were conducted on domains an order of magnitude smaller than those of spatially growing simulations $[6,13]$.

\section{B. Wall-normal velocity profile}

The governing equations in Eqs. (2)-(4) rely only on a single scaling. Because $q$ broadly rescales the entire boundary layer and since the majority of the boundary layer is covered by the outer layer, it can be assumed that $q$ is an outer-scale. This was confirmed in Ruan and Blanquart [5] and $q^{\prime} / q$ was found to be well-approximated by $\theta^{\prime} / \theta$. However, in the near-wall region $\left(x_{2}^{+}<10\right)$, the relevant near-wall length scale is $\delta_{v}=v / \sqrt{\tau_{w} / \rho}$. One might expect the relevant source term to be proportional to $\delta_{v}^{\prime} / \delta_{v}$ rather than $q^{\prime} / q$. One can show that this may lead to a discrepancy in the transpiration velocity profiles of growing and periodic boundary layers.

Consider the ensemble averaged continuity equation and its wall-normal integrated form

$$
\begin{gathered}
\frac{\partial \bar{u}_{2}}{\partial \xi_{2}}=\xi_{2} \frac{q^{\prime}}{q} \frac{\partial \bar{u}_{1}}{\partial \xi_{2}} \\
\bar{u}_{2}\left(\xi_{2}\right)=\int_{0}^{\xi_{2}} \frac{q^{\prime}}{q} \xi_{2}^{*} \frac{\partial \bar{u}_{1}}{\partial \xi_{2}^{*}} d \xi_{2}^{*},
\end{gathered}
$$

where $:$ defines ensemble-averaged quantities. Figure 1 compares the wall-normal velocity profiles between a stream-wise growing [13] and periodic [5] boundary layer at $\operatorname{Re}_{\delta^{*}}=1460$. Both profiles agree very well in the outer layer. The periodic profile overshoots the non-periodic profile in the inner layer and as a direct consequence, overshoots the final free stream value by about $5 \%$. This result is consistent with the fact that the inner layer grows much more slowly than the outer layer and so the broadband use of $q^{\prime} / q$ as a rescaling parameter throughout the boundary layer provides small inaccuracies in the transpiration velocity. A wall-normal varying value of $q^{\prime} / q$ may be necessary to capture appropriately the evolution of $u_{2, \infty}$. Obtaining a wall-normal varying metric source term of $q^{\prime} / q$ would require rescaling the wall-normal coordinate by $q^{m s}\left(x_{1}, x_{2}\right)$ instead of $q\left(x_{1}\right)$. 


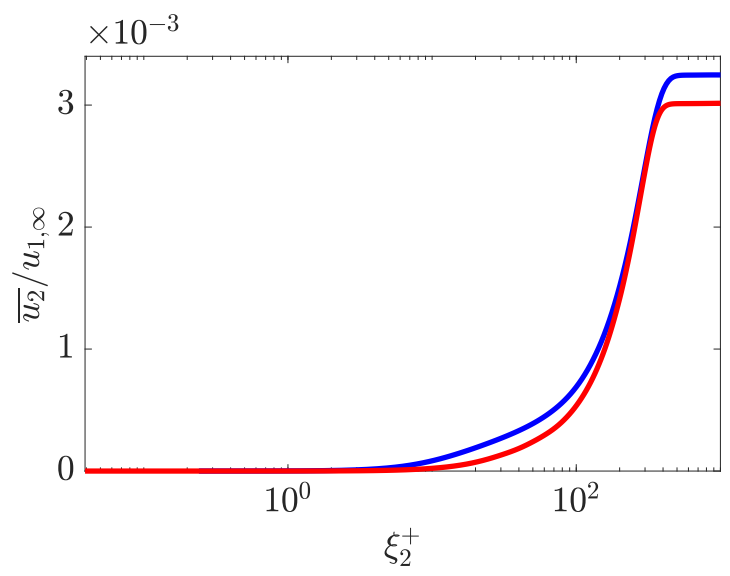

FIG. 1. Profiles of normalized wall-normal velocity $\bar{u}_{2} / u_{1, \infty}$ and for $\operatorname{Re}_{\delta^{*}}=1460$. Colors: (red) streamwise developing DNS [15], (blue) Periodic DNS [5].

\section{A PRIORI MULTISCALE ANALYSIS}

The current section analyzes the multiscale nature of the boundary layer and investigates the impact of multi-scale terms on the mean velocity profile.

\section{A. A priori analytical multicale behavior}

The discussion in the previous section suggested that a rescaling by $q^{m s}=q^{m s}\left(x_{1}, x_{2}\right)$ might be necessary to better capture stream-wise growth effects on the mean flow. For example, after applying a wall-normal rescaling by $q^{m s}$ to $\partial\left\langle u_{1}\right\rangle_{x_{3}, t} / \partial x_{1}$, one would expect the following relation:

$$
\frac{\partial\left\langle u_{1}\right\rangle_{x_{3}, t}}{\partial x_{1}}=-\xi_{2} \frac{q_{x}^{m s}}{q^{m s}} \frac{\partial\left\langle u_{1}\right\rangle_{\xi_{3}, t}}{\partial \xi_{2}} \approx-x_{2} \frac{q_{x}^{m s}}{q^{m s}} \frac{\partial\left\langle u_{1}\right\rangle_{x_{3}, t}}{\partial x_{2}} .
$$

Boundary layer data from spatially growing DNS [13] can be used to evaluate the LHS and RHS of Eq. (7) to extract a profile for $q_{x}^{m s} / q^{m s}$. Figure 2 compares the resulting extracted profiles against

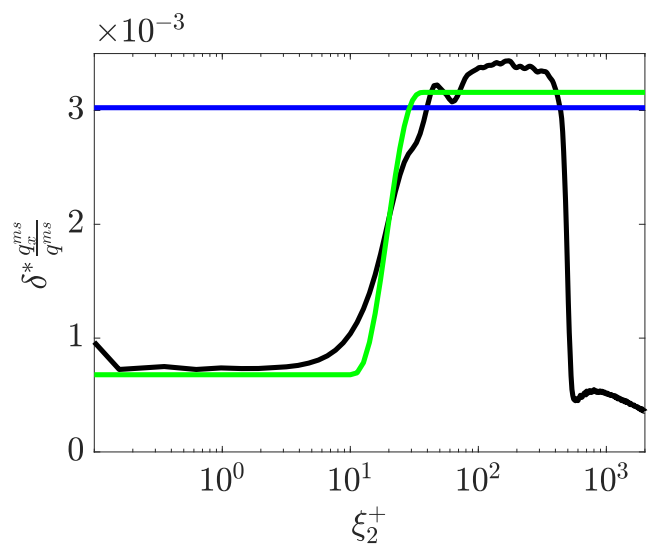

(a)

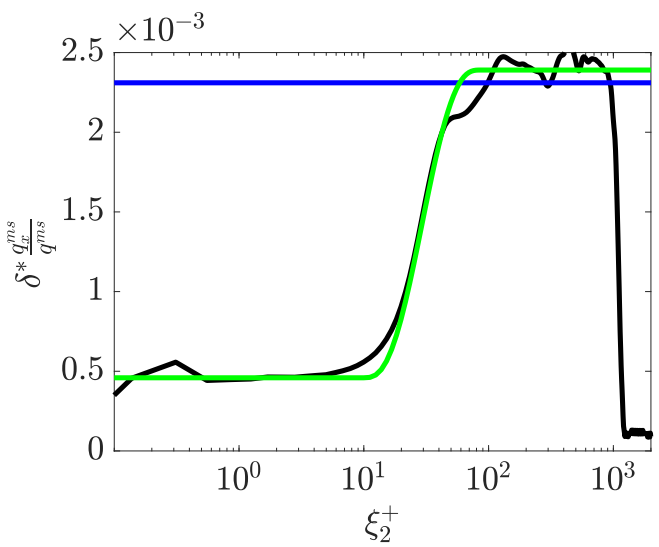

(b)

FIG. 2. Normalized profiles of $q_{x}^{m s} / q^{m s}$ extracted from Ref. [13] for $\operatorname{Re}_{\delta^{*}}=1460$ (a), 3550 (b). Lines: (blue) $\delta^{*} q^{\prime} / q$ [Eq. (4)]; (black) Extracted $\delta^{*} q_{x}^{m s} / q^{m s}$ [Eq. (7)]; (green) Blending $\delta^{*} q_{x}^{m s} / q^{m s}$ [Eqs. (17), (19)]. 
extracted values of $q^{\prime} / q$ using Eq. (4) for two different $\operatorname{Re}_{\delta^{*}}$. It is clear that $q_{x}^{m s} / q^{m s}$ varies throughout the buffer and mesolayer, up to $\xi_{2}^{+} \approx 100$. In both the near-wall region $\left(\xi_{2}^{+}<10\right)$, and in the outer layer $\left(\xi_{2} / \theta \gtrsim 1\right), q_{x}^{m s} / q^{m s}$ is approximately constant.

The near-wall plateau can be understood by invoking the law of the wall $\left(u_{1}^{+} \approx \xi_{2}^{+}\right.$for $\left.\xi_{2}^{+}<10\right)$. The right- hand side of Eq. (7) becomes

$$
\frac{q_{x}^{m s}}{q^{m s}} \xi_{2} \frac{\partial\left\langle u_{1}\right\rangle_{\xi_{3}, t}}{\partial \xi_{2}}=\frac{q_{x}^{m s}}{q^{m s}} \xi_{2}^{+} \frac{\partial\left(u_{1}^{+} u_{\tau}\right)}{\partial \xi_{2}^{+}} \approx \frac{q_{x}^{m s}}{q^{m s}} u_{\tau} \xi_{2}^{+}=\xi_{2} \frac{q_{x}^{m s}}{q^{m s}} \frac{\tau_{w}}{\mu},
$$

where inner scale notation has been used: $u^{+}=\left\langle u_{1}\right\rangle_{\xi_{3}, t} / u_{\tau}, \xi_{2}^{+}=\xi_{2} / \delta_{v}, u_{\tau}=v / \delta_{v}$. A Taylor expansion of the LHS of Eq. (7) around $\xi_{2}=0$ yields

$$
-\frac{\partial\left\langle u_{1}\right\rangle_{\xi_{3}, t}}{\partial x_{1}} \approx-\xi_{2} \frac{\partial}{\partial x_{1}}\left(\left.\frac{\partial\left\langle u_{1}\right\rangle_{\xi_{3}, t}}{\partial \xi_{2}}\right|_{\xi_{2}=0}\right)=-\xi_{2} \frac{\tau_{w}^{\prime}}{\mu} .
$$

Finally, Eq. (7) simplifies to

$$
\left.\frac{q_{x}^{m s}}{q^{m s}}\right|_{\text {inner }} \approx-\frac{\tau_{w}^{\prime}}{\tau_{w}}=-\frac{C_{f}^{\prime}}{C_{f}}=2 \frac{\delta_{v}^{\prime}}{\delta_{v}} .
$$

The outer plateau can be understood by using the law of the wake $\left(u^{+}=U_{\infty}^{+}+f_{o}\left(\xi_{2} / \Delta\right)\right.$, for some universal profile $f_{o}$, where $\Delta=\delta^{*} U_{\infty}^{+}$is the Clauser thickness). The LHS of Eq. (7) gives

$$
-\frac{\partial\left\langle u_{1}\right\rangle_{\xi_{3}, t}}{\partial x_{1}}=-u_{\tau}^{\prime} f_{o}+\frac{\Delta^{\prime}}{\Delta} \frac{\xi_{2}}{\Delta} u_{\tau} f_{o}^{\prime} \approx \frac{\Delta^{\prime}}{\Delta} \frac{\xi_{2}}{\Delta} u_{\tau} f_{o}^{\prime},
$$

where $\left|u_{\tau}^{\prime} / u_{\tau}\right| \ll \Delta^{\prime} / \Delta$ has been used to simplify the equation. The RHS of Eq. (7) also gives

$$
\xi_{2} \frac{q_{x}^{m s}}{q^{m s}} \frac{\partial\left\langle u_{1}\right\rangle_{\xi_{3}, t}}{\partial \xi_{2}}=\frac{q_{x}^{m s}}{q^{m s}} \frac{\xi_{2}}{\Delta} u_{\tau} f_{o}^{\prime} .
$$

Finally, Eq. (7) simplifies to

$$
\left.\frac{q_{x}^{m s}}{q^{m s}}\right|_{\text {outer }} \approx \frac{\Delta^{\prime}}{\Delta}
$$

\section{B. Budget analysis of multiscale behavior}

The following analysis focuses on the effects of employing the wall-normal rescaling by $q^{m s}\left(x_{1}, x_{2}\right)$ on the $u$-momentum equation in contrast to the effects of employing a constant wallnormal rescaling by $q\left(x_{1}\right)$.

The following governing equations are obtained after applying the same two assumptions of statistical homogeneity and a narrow stream-wise domain, and after applying the same order-ofmagnitude simplifications (see Sec. II A):

$$
\begin{gathered}
\frac{\partial u_{i}}{\partial \xi_{i}}=\xi_{2} \frac{q_{x}^{m s}}{q^{m s}} \frac{\partial u_{1}}{\partial \xi_{2}} \\
\frac{\partial u_{i}}{\partial t}=-u_{j} \frac{\partial u_{i}}{\partial \xi_{j}}-\frac{1}{\rho} \frac{\partial P}{\partial \xi_{i}}+v \frac{\partial^{2} u_{i}}{\partial \xi_{j}^{2}}+\xi_{2} \frac{q_{x}^{m s}}{q^{m s}} u_{1} \frac{\partial u_{i}}{\partial \xi_{2}} .
\end{gathered}
$$

An a priori analysis of the stream-wise momentum equation is now conducted. To do so, spanwise and temporal averages are applied to Eq. (15) to obtain

$$
\underbrace{\left\langle u_{j} \frac{\partial u_{i}}{\partial \xi_{j}}\right\rangle_{\xi_{3}, t}}_{\text {convective terms }}=\underbrace{v \frac{\partial^{2}\left\langle u_{i}\right\rangle_{\xi_{3}, t}}{\partial \xi_{j}^{2}}}_{\text {viscous terms }}+\underbrace{\xi_{2}\left\langle\frac{q_{x}^{m s}}{q^{m s}} u_{1} \frac{\partial u_{i}}{\partial \xi_{2}}\right\rangle_{\xi_{3}, t}}_{\text {source term }}
$$




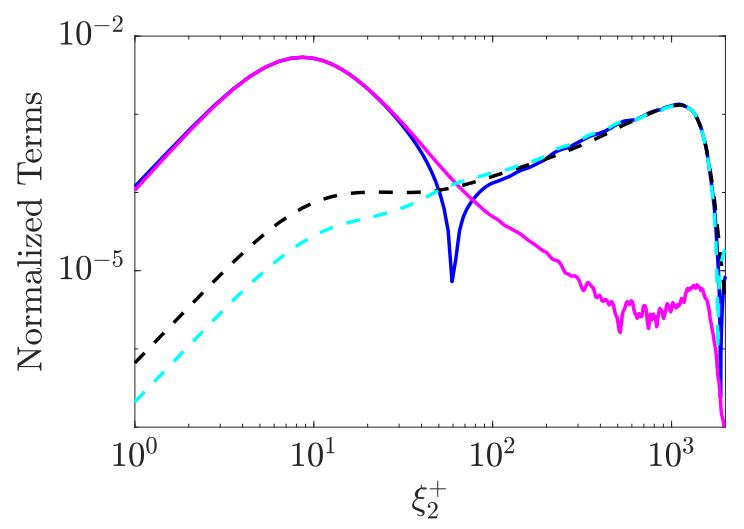

FIG. 3. Streamwise momentum magnitude budget from DNS data (Ref. [13]) at $\operatorname{Re}_{\delta^{*}}=5600$. Lines: (solid blue) Convective terms; (solid magenta) Viscous terms; (dashed black) $\left|\left\langle\frac{q^{\prime}}{q} \xi_{2} u_{1} \frac{\partial u_{1}}{\partial \xi_{2}}\right\rangle_{\xi_{3}, t}\right|$; (dashed cyan) $\left|\left\langle\frac{q_{2}^{m s}}{q^{m s s}} \xi_{2} u_{1} \frac{\partial u_{1}}{\partial \xi_{2}}\right\rangle_{\xi_{3}, t}\right|$.

A wall-normal budget of the stream-wise momentum equation is computed using DNS data [13] at $\operatorname{Re}_{\delta^{*}}=5600$ and the results are shown in Fig. 3. On the same budget, the original source term $\left\langle\xi_{2}\left(q^{\prime} / q\right) u_{1} \partial_{\xi_{2}}\left(u_{1}\right)\right\rangle_{\xi_{3}, t}$ is shown for direct comparison against the new source term from Eq. (16).

The original and new metric source terms agree very well in the outer layer and balance the Reynolds shear stress. As expected, they differ by a factor of $\sim 4$ in the inner layer. However, they both remain orders of magnitudes smaller than the convective and viscous terms. This observation is consistent with Klewicki et al. (2007) who noted that in the near wall region, the viscous and shear stress terms balance nearly completely.

In the near-wall region, the contribution of the multi-scale source term will always remain many orders of magnitude smaller than all other terms, regardless of Reynolds number. This is directly due to the slow growth rate of the flat-plate boundary layer (e.g., in the inner layer the source term is small). It can be shown that the viscous term scales like $u_{\tau}^{2} / \delta_{v}$ in the inner layer. The multiscale metric term scales like $u_{\tau}^{2} q_{x}^{m s} / q^{m s}$. Hence, the ratio of the multiscale metric term to the viscous term scales like $\delta_{\nu} q_{x}^{m s} / q^{m s} \sim \delta_{\nu}^{\prime}$, which is monotonically decreasing with Reynolds number. Similarly, the single-scale metric term scales like $u_{\tau}^{2} q^{\prime} / q$ and its ratio to the viscous term scales like $\delta_{\nu} q^{\prime} / q \approx$ $\delta_{\nu} \theta^{\prime} / \theta \sim C_{f} / \operatorname{Re}_{\tau}$. This ratio is also monotonically decreasing with Reynolds number. In both cases, neither metric source term is significant in the near wall region with respect to the viscous term.

In summary, from an a priori point of view, it appears that implementing a multiscale rescaling function $q_{x}^{m s} / q^{m s}$ should not provide significant benefits on the mean profile of stream-wise velocity. However, an a posteriori perspective is still needed to clarify impacts on other turbulent quantities.

\section{A POSTERIORI ANALYSIS OF MULTISCALE SIMULATIONS}

In this section, simulations are performed using the a model for $q_{x}^{m s} / q^{m s}$. The key purpose of this section is to conduct an a posteriori analysis of multiscale effects.

\section{A. Fitted blending function}

Conducting a posteriori analyses requires imposing a functional form for $q_{x}^{m s} / q^{m s}$ similar to methods used in Ref. [16]. Section III A has shown that near the wall $q_{x}^{m s} / q^{m s} \approx 2 \delta_{v}^{\prime} / \delta_{v}$, and far from the wall, $q_{x}^{m s} / q^{m s} \approx \Delta^{\prime} / \Delta$. To transition smoothly between the two regions, a one-parameter 
TABLE I. DNS parameters for the turbulent boundary layer simulation cases. * indicates simulations taken from Ref. [5].

\begin{tabular}{lcccc}
\hline \hline Dataset & $\mathrm{Re}_{\delta^{*}}$ & Governing equations & $N x \times N y \times N z$ & Sample Time $\delta_{99} / u_{\tau}$ \\
\hline BL1460* & 1460 & Eqs. (2)-(4) & $300 \times 120 \times 160$ & 30 \\
BL3550* & 3550 & Eqs. (2)-(4) & $648 \times 230 \times 338$ & 15 \\
BL1460MS & 1460 & Eqs. (14), (15), (19) & $300 \times 120 \times 160$ & 30 \\
BL3550MS & 3550 & Eqs. (14), (15), (19) & $648 \times 230 \times 338$ & 15 \\
\hline \hline
\end{tabular}

smoothed step function is employed.

$$
\frac{q_{x}^{m s}}{q^{m s}}=\frac{\Delta^{\prime}}{\Delta} g\left(\xi_{2}\right)+2 \frac{\delta_{v}^{\prime}}{\delta_{v}}\left[1-g\left(\xi_{2}\right)\right]=\frac{\Delta^{\prime}}{\Delta}\left[g\left(\xi_{2}\right)+\frac{2\left(1-g\left(\xi_{2}\right)\right)}{\kappa u_{1, \infty}^{+}+1}\right],
$$

where the log-law has been used to relate $\delta_{v}^{\prime} / \delta_{v}$ to $\Delta^{\prime} / \Delta$, the Kármán constant is approximated by $\kappa=0.41$, and $g\left(\xi_{2}\right)$ is a smooth step function defined by

$$
g\left(\xi_{2}\right)= \begin{cases}0 & \xi_{2}^{+} \leqslant 10 \\ 6 r^{5}-15 r^{4}+10 r^{3} & \xi_{2} \in\left[10 \delta_{v}, 0.1 \delta_{99}\right] \\ 1 & \xi_{2} \geqslant 0.1 \delta_{99}\end{cases}
$$

where $r=\ln \left(\xi_{2}^{+} / 10\right) / \ln \left(0.1 \delta_{99} / \delta_{v}\right)$. Here, it has been assumed that the near-wall region extends up to $10 \delta_{v}$, and the wake region extends down to $0.1 \delta_{99}$. Thus, Eq. (17) provides a closure equation for $q_{x}^{m s} / q^{m s}$ in terms of $\Delta^{\prime} / \Delta$.

To complete the closure for $\Delta^{\prime} / \Delta$, the $u$-momentum and continuity equations were integrated in the wall-normal direction and then averaged in the statistically homogeneous directions $\left(\xi_{1}, \xi_{3}\right)$ to provide the following closure equation:

$$
\frac{\tau_{w}}{\rho} \frac{\Delta}{\Delta^{\prime}}=\int_{0}^{\infty}\left[g\left(\xi_{2}\right)+\frac{2\left(1-g\left(\xi_{2}\right)\right)}{\kappa u_{1, \infty}^{+}+1}\right] \xi_{2} \frac{\partial}{\partial \xi_{2}}\left(\left\langle u^{2}\right\rangle_{\xi_{1}, \xi_{3}, t}-\langle u\rangle_{\xi_{1}, \xi_{3}, t} u_{1, \infty}\right) d \xi_{2} .
$$

Overall, Eqs. (17)-(19) provide a fitted function to the actual $q_{x}^{m s} / q^{m s}$ extracted via Eq. (7). A comparison between the fitted and extracted profiles of $q_{x}^{m s} / q^{m s}$ is shown in Fig. 2 for $\operatorname{Re}_{\delta^{*}}=1460$ and 3550. The fit agrees very well with the extracted function in both the outer layer plateau all the way down to $\xi_{2}^{+}=10$. There is a $10 \%$ relative error in the outer layer plateau value for the lower Reynolds number comparison, but only a 3\% relative error for the higher Reynolds number comparison. The inner layer plateau is also within 5\%. This difference is primarily due to the use of the log-law for low Reynolds number flows and these differences are expected to decrease with increased Reynolds number.

It should be noted that Eq. (19) will be solved in real time for simulation purposes. Thus, the governing equations are completely closed.

\section{B. Simulation parameters and numerical methods}

The following cases were simulated and are summarized in Table I. Cases BL1460MS and BL3550MS solve Eqs. (14) and (15) with closure Eq. (19). All cases have periodic span-wise and streamwise boundary conditions, and have nonperiodic wall-normal directions. The bottom of the domain has a no-slip boundary condition, and a Neumann boundary condition is applied to the top of the computational domain. Mass conservation is conducted at the wall-normal outlet. Wall-normal integration of Eqs. (2) and 14 directly shows that any closure for $q^{\prime} / q$ and $q_{x}^{m s} / q^{m s}$, respectively, provides a value for $u_{2, \infty}$. Closure equations Eqs. (4) and (19) are evaluated instantaneously at each time step of the simulation. 


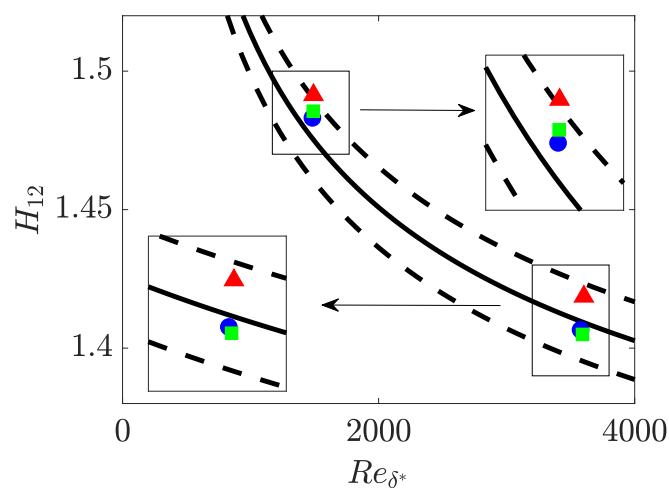

(a)

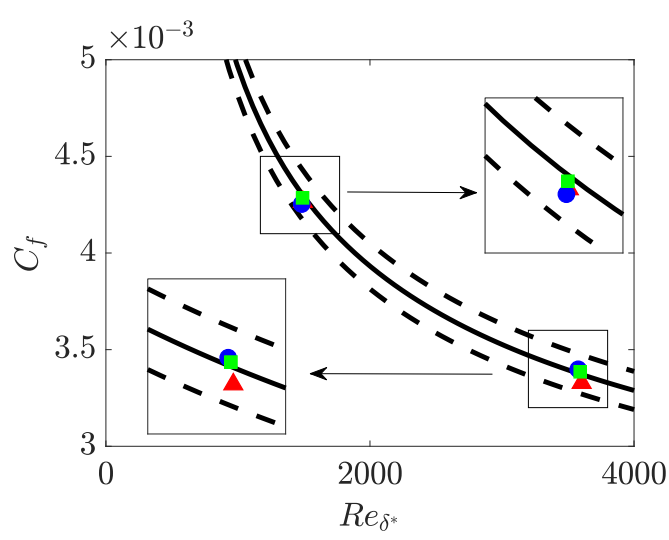

(b)

FIG. 4. (a) Shape factor $H_{12}$ as a function of Reynolds number $\operatorname{Re}_{\delta^{*}}$. Solid line represents empirical fit by Ref. [3], and dashed lines indicate $\pm 1 \%$. (b) Skin-friction as a function of $\operatorname{Re}_{\delta^{*}}$. Solid line represents the extended Coles-Fernholz relation with $\kappa=0.384, C=3.3, D_{0}=182, D_{1}=-2466$ [14]. Dashed lines indicate $\pm 3 \%$. Symbols: $\triangle$ (red) DNS [13]; $\square$ (green) Cases BL1460MS and BL3550MS (DNS); $\circ$ (blue) Cases BL1460 and BL3550 (DNS) [5].

The domain size, $\left(L_{x}, L_{y}, L_{z}\right)$, is determined primarily by the sizes of large-scale motions (LSMs). Pressure fluctuations can extend to wall-normal heights of $2.4 \delta_{99}$ [11], setting the minimum requirement for wall-normal height. Consequently, we set our domain height to $18 \delta^{*} \sim 3 \delta_{99}$. We opt for a span-wise width of $14 \delta^{*} \sim 2.5 \delta_{99}$, since low-momentum streaks are approximately $0.5 \delta_{99}$ in width $[7,17]$. Finally, the LSMs corresponding to bulges or hairpin packets have a maximum stream-wise length of $3 \delta_{99}$ [18-22]; we opt for a domain of $7 \delta_{99}$ in stream-wise length. BL1460MS and BL3550MS have domain sizes identical to cases BL1460 and BL3550 in Ruan and Blanquart [5]. In general, the very-large-scale motions tend to have sizes much larger than the current simulation domain size [19]. However, as has been argued in shorter channel flow simulations Refs. [23,24], these structures still exist in these smaller domains, and are just aliased onto smaller turbulent structures. For channel flow simulations, they ultimately have minimal effects on one-point statistics $[23,24]$.

The resolution is chosen so that the smallest turbulent structures can be resolved adequately. The stream-wise and span-wise grids are uniform with $\Delta \xi_{1}^{+}=9$ and $\Delta \xi_{3}^{+}=6$, which is comparable to the resolution parameters of Orlu etal. [13] $\left(\Delta \xi_{1}^{+} \approx 8.5, \Delta \xi_{3}^{+} \approx 4\right)$. The wall-normal domain uses a hyperbolic stretching with eight points in the viscous sublayer, $\left(\xi_{2}^{+}<5\right)$, with $\Delta \xi_{2, \min }^{+} \approx 0.3$. This is comparable to the wall-normal resolution of Orlu et al. [13] who had 10 points in the viscous sublayer at their lowest Reynolds number.

The governing equations are solved using NGA [25]. The numerical code solves the conservative-variable formulation of the low-Mach Navier-Stokes equations with staggered finite difference operators. It uses a fractional step method to enforce continuity and is fully secomd order in time and second order in space.

\section{Simulation results}

Figure 4 compares calculated skin-friction coefficients and shape factors from simulations BL1460MS and BL3550MS against those from single-scale simulations values [5], growing simulations [13], and empirical fits [3,14]. The multiscale simulations have shape factors that are within $0.1 \%$ of the single-scale values and are within $0.4 \%$ of the stream-wise growing DNS values. The multiscale skin-friction coefficients are within $0.6 \%$ of the single-scale values and are within $2 \%$ of the stream-wise growing DNS values. The multiscale simulation shape factor and 


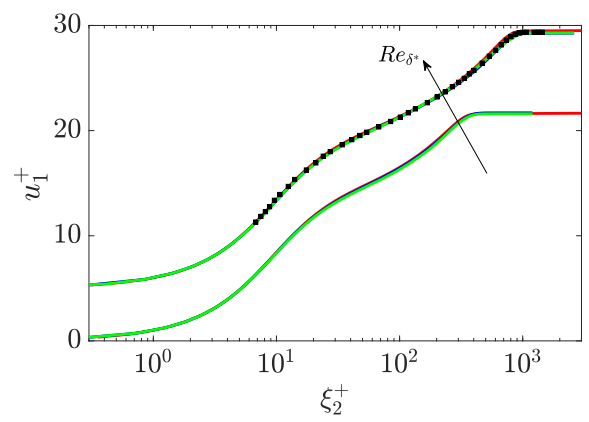

(a)

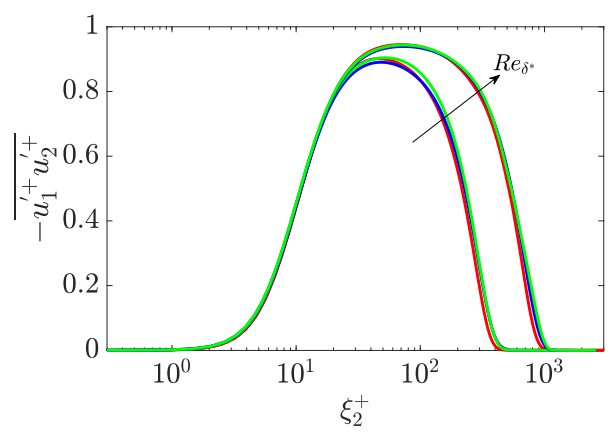

(c)

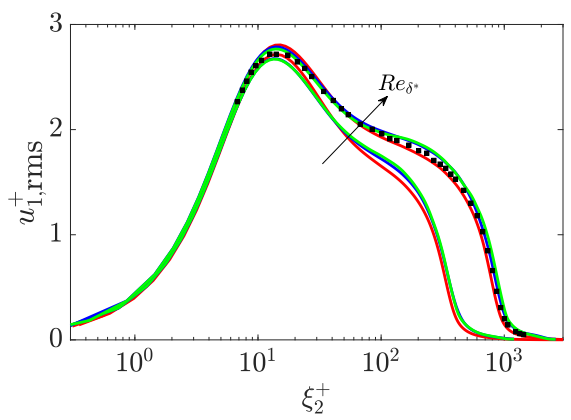

(b)

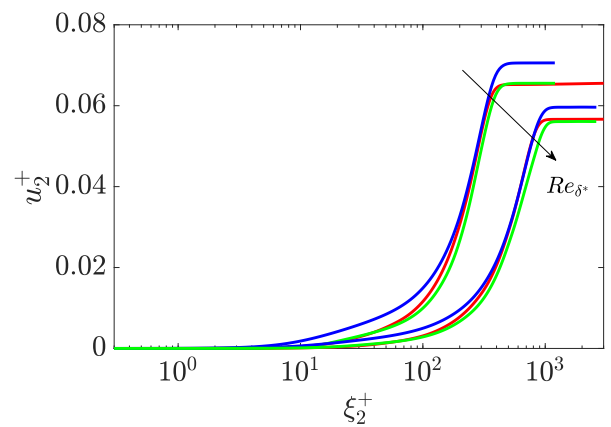

(d)

FIG. 5. (a) $u_{1}^{+}$(b) $u_{1, \mathrm{rms}}^{+}$(c) $-\overline{u_{1}^{\prime+} u_{2}^{\prime+}}$ (d) $u_{2}^{+}$vs $\xi_{2}^{+}$for $\operatorname{Re}_{\delta^{*}}=1460$, 3550. Legend: (red) DNS [13]; (blue) Single-scale cases BL1460, BL3550 [5]; (green) Multiscale DNS cases BL1460MS and BL3550MS; $\square$ (black) [13] experimental data.

skin-friction coefficient both remain well within $0.5 \%$ and $2.2 \%$ of the corresponding empirical values, respectively. More importantly, the differences between the single-scale and multiscale simulation results are significantly less than between either and the growing simulation results.

Figure 5 shows the inner-scaled mean stream-wise velocity profiles of the different Reynolds number simulation results in contrast with those of Ref. [13], which had a stream-wise growing boundary layer, and the single-scale simulation results of Ruan and Blanquart [5]. The good agreement of shape factors and skin-friction coefficients directly implies good agreement of the mean stream-wise velocity profiles for both $\operatorname{Re}_{\delta^{*}}=1460$ and 3550. The normalized Reynolds stress and rms profiles are also plotted in Fig. 5. The near-wall peak at $\xi_{2}^{+} \approx 15$ and the general good agreement in the wake confirm that the multiscale rescaling effects appear to be minimal on both the measured quantities of mean stream-wise velocity and rms. Differences in the mean wall-normal velocity profile do have an impact on the behavior of the Reynolds shear stress and second-order statistics. In the mean stream-wise momentum balance, an increased source term directly corresponds to an increased Reynolds shear stress. Similar conclusions have been pointed out in Ref. [26]. The differences in secondorder statistics are within 5\% across the boundary layer, and the impact appears to decrease with increasing Reynolds number. Overall, the multiscale variance profiles are consistently larger than their single-scale counterparts.

Figure 5(d) shows the wall-normal velocity profiles. There is marked improvement in both the near-wall behavior and free-stream value. Specifically, $u_{2, \infty}^{+}$varies by about $5 \%$ between the multiscale and single-scale cases, and the near wall behavior has a relative deviation of $3 \%$ between the multiscale and single-scale cases as well. The deviation between the single-scale simulation and multiscale simulation decreases with increasing Reynolds number. From Fig. 5, it can be extrapolated that with the multiscale correction, the mean wall-normal velocity profile decreases 
in magnitude, which corresponds to an increase in magnitude of the second-order statistics. Asymptotically, as $\operatorname{Re}_{\tau} \rightarrow \infty$, the inner layer vanishes, and thus the wall-normal region where $q_{x}^{m s} / q^{m s} \ll q^{\prime} / q$ is small. Consequently, as the mean wall-normal velocity profile is an integration of $q_{x}^{m s} / q^{m s} \xi_{2} \partial \bar{u}_{1} / \partial \bar{\xi}_{2}$, the differences between the multi-scale and single scale $\bar{u}_{2}$ are expected to vanish asymptotically.

The variance and wall-normal velocity profiles are dependent on the choice of the parameters fit used in Eq. (17). Specifically, the value of $q_{x}^{m s} / q^{m s}$ in the near-wall region is inversely proportional to the value of $\kappa$. Consequently, with increasing $\kappa$, the source term in continuity is increased in magnitude in the inner layer and thus, following arguments made in Sec. II B, the wall-normal velocity profile in the outer layer and the overall transpiration velocity will be larger in magnitude. Based on Fig. 5, increases in mean wall-normal velocity profiles correspond to decreases in secondorder statistics magnitude. Overall, with increasing values of $\kappa$, one expects decreasing magnitudes of the second-order statistics.

\section{DISCUSSION OF REYNOLDS NUMBER CONTRIBUTION}

\section{A. Reynolds number dependence}

Classical descriptions of the boundary layer require two different scalings for the inner and outer layers [27]. To avoid the controversy of suggesting what velocity and length scales are involved $[28,29]$, we will appeal to analysis via nondimensional groups. For flat plate boundary layers, the ensemble-averaged mean stream-wise velocity is given by

$$
\frac{\bar{u}_{1}}{u_{1, \infty}}=f\left(\frac{x_{2}}{q}, \operatorname{Re}_{\mathrm{q}}\right) .
$$

Consequently, the stream-wise derivative is given by

$$
\frac{\partial \bar{u}_{1}}{\partial x_{1}}=\underbrace{-x_{2} \frac{q^{\prime}}{q} \frac{\partial \bar{u}_{1}}{\partial x_{2}}}_{\text {source term }}+\underbrace{\frac{q^{\prime}}{q} \operatorname{Re}_{\mathrm{q}} \frac{\partial \overline{\mathrm{u}}_{1}}{\partial \operatorname{Re}_{\mathrm{q}}}}_{\text {error term }} .
$$

Note that the last term in Eq. (21) is omitted in Eq. (2) as a consequence of Assumption 1. Correspondingly, Eq. (2)-(4) lack any Reynolds number partial terms. Note that the neglecting of explicit Reynolds number partial derivative terms does not remove the Reynolds number dependence of the mean quantities, i.e., the viscous terms still provide an implicit dependence on the Reynolds number. The evolution of the shape and skin-friction coefficient (see Fig. 4) are illustrative examples of this Reynolds number dependence. The good agreement of the global quantities intuitively implies that the exclusion of the Reynolds number partial terms has a negligible impact on the mean stream-wise velocity for the current range of Reynolds numbers. However, it is unclear if this result persists for high $\operatorname{Re}_{\delta^{*}}$.

The "error" term is a consequence of zero pressure gradient boundary layer flow relying on two distinct length-scales. Specifically, the second key, near-wall, non-dimensional group is $\operatorname{Re}_{q}$. For exactly parallel boundary layer flow (sink flow), the flow is dependent exactly on a single-length scale: $\delta_{99}$. In this case, the imposed nondimensionalized pressure gradient is the key nondimensional group. In this case, the "error" term is exactly zero and the HNSE exactly follow sink flow behavior provided appropriate boundary conditions and pressure gradient terms are retained.

The objective is to determine the magnitude of this "error" term over a large range of Reynolds numbers and compare its magnitude to that of the source term. First, we start by recognizing that the source term in the continuity equation and in Eq. (21) is nothing more than the log-indicator function $\Psi=x_{2} \partial \bar{u}_{1} / \partial x_{2}$ rescaled by $q^{\prime} / q$. It can be computed easily from any DNS data. Figure 6(a) presents the rescaled source term calculated from various DNS and experimental databases $[5,6,13]$ for $\operatorname{Re}_{\theta}=4000$. All curves feature two peaks: one in the inner and one in the outer layer. Overall, 


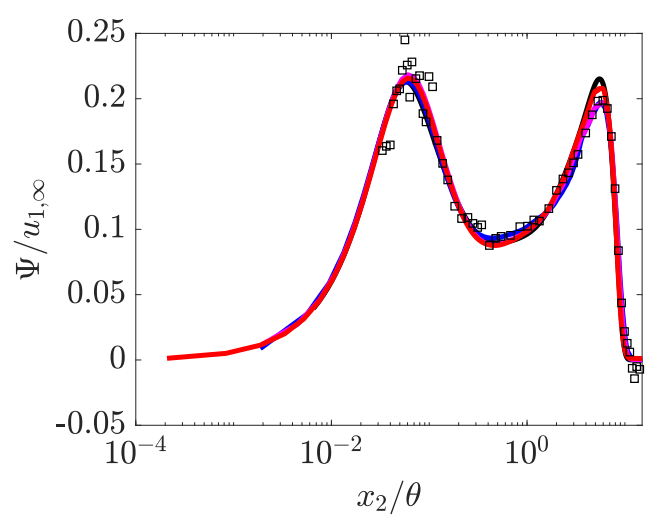

(a)

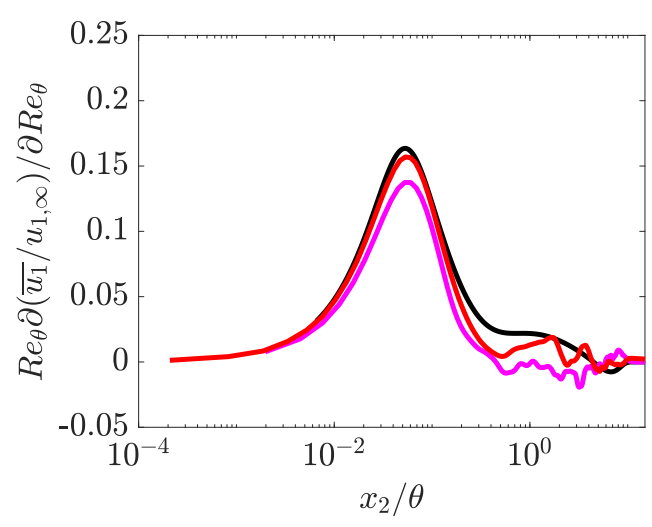

(b)

FIG. 6. (a) Scaled source term $\Psi / U_{\infty}=x_{2} \partial\left(\bar{u}_{1} / u_{1, \infty}\right) / \partial x_{2}$ (b) "error" term $\operatorname{Re}_{\theta} \partial\left(\overline{\mathbf{u}}_{1} / \mathrm{u}_{1, \infty}\right) \partial \operatorname{Re}_{\theta}$ at $\operatorname{Re}_{\theta} \approx$ 4000. Symbols indicate experiments. Colors: (black) Composite fit at $\operatorname{Re}_{\theta} \approx 4000$. [3]; (magenta) [6]; (blue) [5]; (red) [13].

there is good agreement between experimental and DNS profiles, with the experimental values featuring scatter near the wall due to resolution [13].

The "error" term can be extracted from stream-wise growing boundary layers using Eq. (21). Unfortunately, experimental data for this particular quantity are not available. Similarly, the "error term" is also not immediately accessible from single-scale periodic simulations. The resulting profiles are shown in Fig. 6(b). Both profiles agree on a near-wall peak and its location, and show only small deviations on its magnitude. Further from the wall, the extracted profiles are close to zero and are very noisy. From Eq. (21), the low magnitude of the "error" term in the outer layer indicates that the majority of the stream-wise variation of $\overline{u_{1}}$ is captured by the source term. Since $q \approx \theta$ is an outer scale, and since the source term describes the growth of the boundary layer due to the growth of $q$, it is expected that the source term should capture most of the stream-wise variation of the outer layer. In contrast, the relatively large magnitude of the "error" term in the inner layer indicates that the majority of the stream-wise variation of $\overline{u_{1}}$ in the inner layer is not captured by the source term. This is consistent with the results shown in Fig. 2. It was found that the metric term $q^{\prime} / q$ overshoots the actual value of $2 \delta_{\nu}^{\prime} / \delta_{\nu}$. Because the source term and "error" term have opposite signs in Eq. (21), it can be understood that in the inner layer, the source term overestimates the actual stream-wise variation and the "error" term accounts for the overshoot. One could conclude that the effect of the "error" term is primarily concentrated in the inner layer.

To extrapolate these results to higher Reynolds numbers, we turn to a composite fit [3]. The $\log$ indicator function is extracted from composite fit profiles and compared to the experimental and numerical profiles. There is good agreement throughout the inner and outer layers. The composite fit overshoots the outer layer peak magnitude by $\sim 5 \%$, and this may be due to low-Reynolds number effects. Overall, the composite fit agrees with experimental and DNS results quite satisfactorily. Similarly, the composite fit is used to compute the "error" term and the resulting profile is shown in Fig. 6(b). Once again, the profile agrees with the extracted DNS profiles on the location of the inner peak at $x_{2} / \theta \approx 0.1$. They only differ in the peak value by $16 \%$.

The "error" term is plotted over a range of Reynolds numbers in Fig. 7(a). The magnitude of the term decreases slowly with increasing Reynolds number (a factor of 2 from $\operatorname{Re}_{\theta}=10^{3}$ to $\operatorname{Re}_{\theta}=10^{8}$ ) and the peak appears to be fixed within the inner layer. Consequently, it will be admittedly difficult to completely capture the mean streamwise velocity gradient $\partial \bar{u}_{1} / \partial x_{1}$ in a point-wise fashion. However, in Sec. V B, we will explore how this error term affects wall-normal 


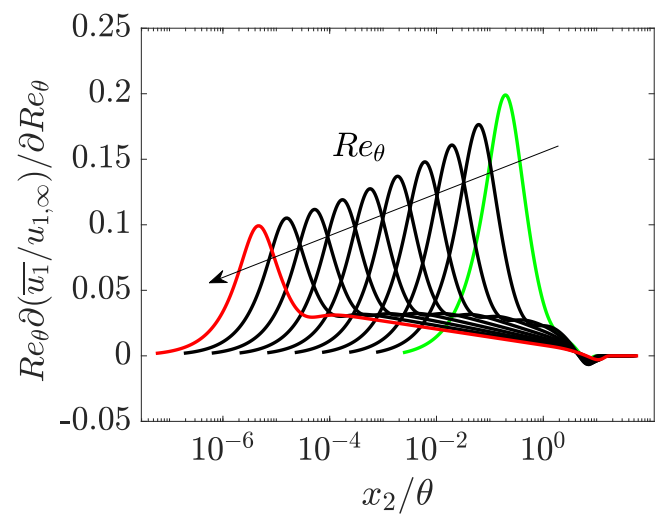

(a)

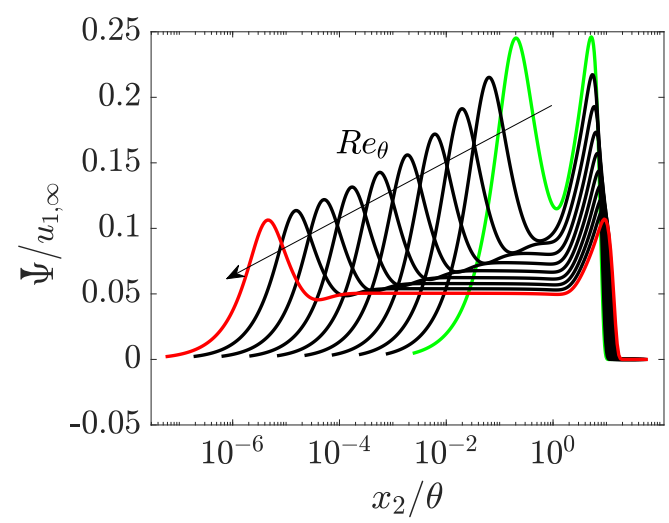

(b)

FIG. 7. (a) "Error" term $\operatorname{Re}_{\theta} \partial\left(\overline{\mathrm{u}}_{1} / \mathrm{u}_{1, \infty}\right) \partial \operatorname{Re}_{\theta}$, and (b) scaled source term $\Psi / u_{1, \infty}=x_{2} \partial\left(\bar{u}_{1} / u_{1, \infty}\right) / \partial x_{2}$, predicted with the composite fit [3], for a range of $\operatorname{Re}_{\theta}=10^{3}-10^{8}$.

integrated quantities. For comparison, the source term contribution is plotted in Fig. 7(b) for the same series of Reynolds numbers.

\section{B. Global quantities}

The previous Reynolds number dependence of the mean velocity profiles can be expressed by analyzing the differences between the solutions to the original Navier-Stokes equations and the solutions to Eqs. (2)-(4). One can equivalently subtract the ensemble average of Eq. (2) from Eq. (21) and evaluate at $x_{1}=x_{0}$ to obtain

$$
x_{2} \frac{\partial\left(\bar{u}_{1}-\bar{u}_{1}^{*}\right)}{\partial x_{2}}-\frac{q}{q^{\prime}} \frac{\partial\left(\bar{u}_{2}-\bar{u}_{2}^{*}\right)}{\partial x_{2}}=\left.\frac{\partial \bar{u}_{1}}{\partial \operatorname{Re}_{\mathrm{q}}}\right|_{x_{0}} \operatorname{Re}_{\mathrm{q}},
$$

where $q^{\prime} / q$ has been divided out and asterisks denote solutions to the transformed governing equations. Stated differently, the difference between the solutions to the Cartesian $\left(\bar{u}_{1}, \bar{u}_{2}\right)$ and transformed governing equations $\left(\bar{u}_{1}^{*}, \bar{u}_{2}^{*}\right)$ is given by the Reynolds number derivative of the mean velocity profile. Equation (22) can now be used to investigate the impact of this "error" term onto global quantities like the skin-friction coefficient and shape factor.

Integrating Eq. (22) in the wall-normal direction and dividing by $\theta$ gives

$$
\underbrace{H_{12}-H_{12}^{*}}_{H_{12} \text { Error }}-\frac{1}{q^{\prime} u_{1, \infty}}\left(u_{2, \infty}-u_{2, \infty}^{*}\right)=\left.\int_{0}^{\infty} \operatorname{Re}_{\theta} \frac{\partial \overline{u_{1}} / u_{1, \infty}}{\partial \operatorname{Re}_{\theta}}\right|_{x_{0}} d\left(\frac{x_{2}}{\theta}\right)=\underbrace{-\operatorname{Re}_{\theta} \frac{\partial \mathrm{H}_{12}}{\partial \operatorname{Re}_{\theta}}}_{\text {"Error" Term }} .
$$

To leading order of $\ln \left(\operatorname{Re}_{\theta}\right)$, the shape factor in the limit of large Reynolds number is approximated by $H_{12}-1 \sim 7.11 \kappa \ln \left(\operatorname{Re}_{\theta}\right)^{-1}$ [14]. The RHS of Eq. (23) can then be approximated as

$$
-\operatorname{Re}_{\theta} \frac{\partial \mathrm{H}_{12}}{\partial \operatorname{Re}_{\theta}} \sim 7.11 \kappa \ln \left(\operatorname{Re}_{\theta}\right)^{-2} .
$$

For cases BL1460 and BL3550, this "error" term is nearly $6 \%$ of the shape factor value. Since the relative error in shape factors from the single-scale simulations [5] was less than $1 \%$, one can conclude that the majority of the "error" term is instead balanced by the second term on the LHS of Eq. (23). This second term describes a difference in transpiration velocities. For cases BL1460 and BL3550, it can be seen from Fig. 5(d) that the transpiration velocities of the single-scale simulations was greater than that of the growing simulation by at most $5 \%$. The error in the transpiration velocity 
to leading order of Re monotonically decays with the log of the Reynolds number. For experimental Reynolds numbers, the agreement in shape factor is only expected to improve.

\section{CONCLUSION}

In this work, we investigated the transpiration velocity of single-scale periodic simulations. Unlike other simulations of turbulent boundary layers, the single-scale periodic boundary layer simulation does not impose a transpiration velocity. In this way, the transpiration velocity characterizes the numerical error in the stream-wise periodic simulation. More specifically, after integrating continuity, the transpiration velocity error was primarily caused by a difference in inner and outer layer growth rates. A second stream-wise periodic numerical framework was formulated to account for the differing layer growth rates. Doing so required generating a fit to a smooth transition from $2 \delta_{v}^{\prime} / \delta_{v}$ in the inner layer to $\Delta^{\prime} / \Delta$ in the outer layer. Under this framework, we verified that the transpiration velocity improved from at most $5 \%$ to $1 \%$ but the remaining mean and turbulent intensities were virtually unchanged. The stream-wise periodic simulations appears insensitive to the transpiration velocity, and the "improved" numerical framework may be superfluous.

Finally, through an error budget of the mean continuity equation, the errors in the mean continuity equation must manifest either as errors in the shape factor or as errors in the transpiration velocity. Based on low Reynolds number results, the former error is orders of magnitude smaller than the latter error. Furthermore, using a composite fit [3], it can be shown that this error vanishes with increasing Reynolds number. Thus it can be concluded that for larger and more practical Reynolds numbers, the use of single-scale stream-wise periodic simulations remains robust.

[1] M. de Silva, N. Hutchins, and I. Marusic, Uniform momentum zones in turbulent boundary layers, J. Fluid Mech. 786, 309 (2015).

[2] N. Hutchins and I. Marusic, Evidence of very long meandering features in the logarithmic region of turbulent boundary layers, J. Fluid Mech. 579, 1 (2007).

[3] P. A. Monkewitz, K. A. Chauhan, and H. M. Nagib, Self-consistent high-Reynolds-number asymptotics for zero-pressure-gradient turbulent boundary layers, Phys. Fluids 19, 115101 (2007).

[4] W. J. Baars, N. Hutchins, and I. Marusic, Reynolds number trend of hierarchies and scale interactions in turbulent boundary layers, Philos. Trans. R. Soc. A 375, 20160077 (2017).

[5] J. Ruan and G. Blanquart, Direct numerical simulations of a statistically stationary streamwise periodic boundary layer via the homogenized Navier-Stokes equations, Phys. Rev. Fluids 6, 024602 (2021).

[6] J. A. Sillero, J. Jiménez, and R. D. Moser, One-point statistics for turbulent wall-bounded flows at reynolds numbers up to 2000, Phys. Fluids 25, 105102 (2013).

[7] J. H. Lee and H. J. Sung, Very-large-scale motions in a turbulent boundary layer, J. Fluid Mech. 673, 80 (2011).

[8] T. S. Lund, X. Wu, and K. D. Squires, Generation of turbulent inflow data for spatially-developing boundary layer simulations, J. Comput. Phys. 140, 233 (1998).

[9] T. Wei and J. Klewicki, Scaling properties of the mean wall-normal velocity in zero-pressure-gradient boundary layers, Phys. Rev. Fluids 1, 082401(R) (2016).

[10] P. Kumar and K. Mahesh, Simple model for mean stress in turbulent boundary layers, Phys. Rev. Fluids 6, 024603 (2021).

[11] M. P. Simens, J. Jiménez, S. Hoyas, and Y. Mizuno, A high-resolution code for turbulent boundary layers, J. Comput. Phys. 228, 4218 (2009).

[12] P. Schlatter, R. Örlü, Q. Li, G. Brethouwer, J. H. M. Fransson, A. V. Johansson, P. H. Alfredsson, and D. S. Henningson, Turbulent boundary layers up to $\mathrm{Re}=2500$ studied through simulation and experiment, Phys. Fluids 21, 051702 (2009).

[13] R. Örlü and P. Schlatter, Comparison of experiments and simulations for zero pressure gradient turbulent boundary layers at moderate Reynolds numbers, Exp. Fluids 54, 1547 (2013). 
[14] K. A. Chauhan, P. A. Monkewitz, and H. M. Nagib, Criteria for assessing experiments in zero pressure gradient boundary layers, Fluid Dynamics Res. 41, 021404 (2009).

[15] R. Örlü and P. Schlatter, Inflow length and tripping effects in turbulent boundary layers, J. Phys.: Conf. Ser. 318, 022018 (2011).

[16] P. Spalart and A. Leonard, Direct Numerical Simulation of Equilibrium Turbulent Boundary Layers, in Turbulent Shear Flows 5 (Springer, Berlin, 1987), pp. 234-252.

[17] C. Tomkins and R. Adrian, Spanwise structure and scale growth in turbulent boundary layers, J. Fluid Mech. 490, 37 (2003).

[18] V. Priymak and T. Miyazaki, Long-wave motions in turbulent shear flows, Phys. Fluids 6, 3454 (1994).

[19] K. C. Kim and R. J. Adrian, Very large-scale motion in the outer layer, Phys. Fluids 11, 417 (1999).

[20] J. C. del Alamo, J. Jiminez, P. Zandonade, and R. D. Moser, Scaling of the energy spectra of turbulent channels, J. Fluid Mech. 500, 135 (2004).

[21] M. Guala, S. E. Hommena, and R. J. Adrian, Large-scale and very-large-scale motions in turbulent pipe flow, J. Fluid Mech. 554, 521 (2006).

[22] B. Balakumar and R. Adrian, Large- and very-large-scale motions in channel and boundary-layer flows, Philos. Trans. R. Soc. A 365, 665 (2007).

[23] O. Flores and J. Jiménez, Hierarchy of minimal flow units in the logarithmic layer, Phys. Fluids 22, 071704 (2010).

[24] A. Lozano-Durán and J. Jiménez, Effect of the computational domain on direct simulations of turbulent channels up to $\operatorname{Re}_{\tau}=4200$, Phys. Fluids 26, 011702 (2014).

[25] O. Desjardins, G. Blanquart, G. Balarac, and H. Pitsch, High order conservative finite difference scheme for variable density low mach number turbulent flows, J. Comput. Phys. 227, 7125 (2008).

[26] X. Chen, F. Hussain, and Z. She, Non-universal scaling transition of momentum cascade in wall turbulence, J. Fluid Mech. 871, R2 (2019).

[27] D. Coles, The law of the wake in the turbulent boundary layer, J. Fluid Mech. 1, 191 (1956).

[28] W. George, Is there a universal log law for turbulent wall-bounded flows? Philos. Trans. R. Soc. A 365, 789 (2007).

[29] M. Jones, T. Nickels, and M. Marusic, On the asymptotic similarity of the zero-pressure-gradient turbulent boundary layer, J. Fluid Mech. 616, 195 (2008). 